\title{
Structural materials: What can we learn from nature?
}

\author{
David Taylor \\ Trinity Centre for Bioengineering, Trinity College Dublin, Ireland
}

\begin{abstract}
The mechanical properties of materials are of vital importance in the natural world. Over millions of years of evolution, Nature has created materials capable of resisting mechanical forces, in our bodies and in those of other animals and plants. Throughout history human beings have created new materials; in particular many materials developed over the last 100 years have greatly surpassed natural materials in their mechanical properties and durability. So is there anything which Nature can still teach us about making and maintaining structural materials? This talk will consider some of the "tricks" which Nature uses, such as bottom-up fabrication processes, functionally graded structures and materials with the capacity for continual self-monitoring and repair. It will be shown that some of these tricks can be used by materials scientists, but some aspects of the behaviour of natural materials are not suitable for copying into manmade structures.
\end{abstract}

\section{NATURE'S MATERIALS ARE NOT VERY GOOD}

From a mechanical point of view, the properties of natural, biological materials are quite poor. Any rational comparison on the basis of properties such as strength, toughness, ductility or energy absorption shows that engineering materials developed in the $20^{\text {th }}$ century - especially metal alloys and fibre composites - far outperform natural materials. So do we really have anything to learn from nature? I believe we do, because (thanks to evolution) nature has been able to make the best possible use of the rather poor-quality materials available. By looking at how nature optimises material use we can learn lessons that we can apply to future materials development.

\section{MATERIAL VERSUS STRUCTURE}

Figure 1 shows a beautiful picture taken by Mueller et al (2006) of a section cut through a tree, revealing the structure near a branch. This image reveals several important aspects of natural materials which are worthy of consideration. Firstly, the material is essentially a fibre composite, in which the fibres have been arranged to alter their orientations in the vicinity of the joint between the main trunk and the side branch to achieve a smooth transition and follow lines of force. This is very difficult (though not impossible) for us to achieve in engineering structures made from fibre composites. This illustrates an important fact which recurs often in natural materials: there is no clear distinction between the material and the structure. Material properties gradually change from place to place within the structure, improving overall performance (e.g. stiffness/weight) and eliminating sudden transitions which are always points of weakness. Similar structure/material interactions can be found in the human body, for example at bone/tendon junctions and in cartilage. Our attempts to replicate this kind of transition, for example using functionally graded materials, is beginning to bear fruit, but we have a long way to go.
The second feature of interest in figure 1 is that the insertion of the branch does not occur only on the surface but goes deep into the middle of the trunk. This happens naturally because the tree and branch have both grown, gradually increasing in size by surface deposition over many years. This is a nice way to achieve a good connection, from which we might learn some lessons useful in engineering structures made by additive manufacturing. However, it also imposes some limitations on the structure: for example, a hollow tube would have a better strength/weight ratio but cannot be made by this process. Some plants (e.g. bamboo) make use of a hollow tube design, which requires a very different growth mechanism.

Thirdly, note the shape of the upper and lower surfaces near the trunk/branch junction (which I have highlighted with thick red lines). These curves are quite complex in form, not the simple fillet radii which an engineering designer would normally use. As Mattheck has shown very elegantly, stress concentration in the region of the joint can be greatly reduced (in fact it can be almost completely eliminated) by relatively subtle changes in curvature in this region (Mattheck 1998).

\section{HIERARCHICAL MATERIAL STRUCTURE}

Natural materials often display structure at several different levels, from the nanostructure of molecular arrangements (e.g. the triple helix of collagen) through various levels up to microstructure (e.g. the aggregation of collagen molecules to form oriented fibrils and fibres at the micron level) and beyond this from the micro to the macro levels (e.g. osteons in bone and the spongy, porous structure of trabecular bone).

Why do these hierarchical levels of structure exist? The simple answer is because natural materials and made "from the bottom up" using the process which we now call Additive Manufacturing. Molecules are created inside cells and gradually assembled into larger structural units. We are learning how to do this for engineering materials, and there have been great advances in nanotechnology, but we have 


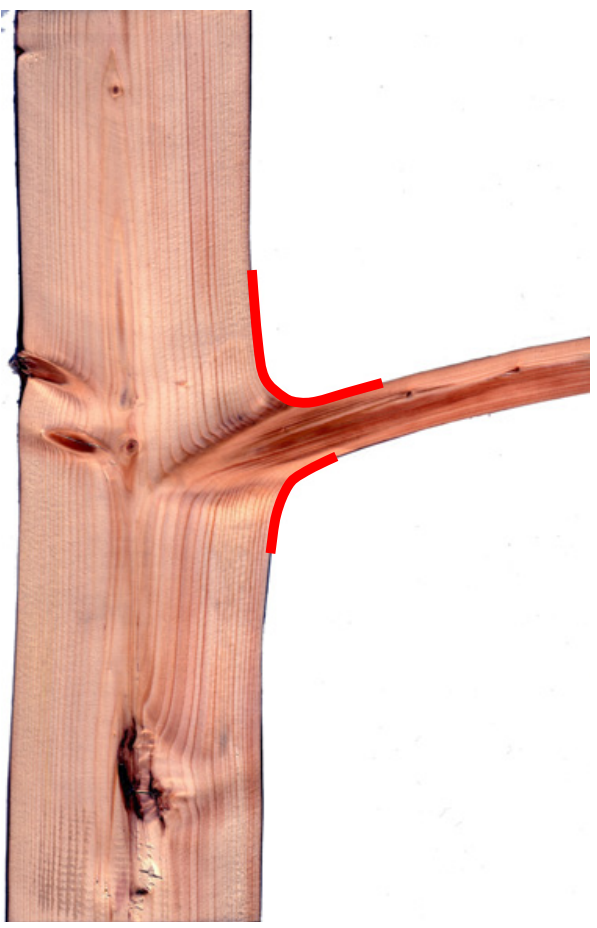

Figure 1. Longitudinal section through a tree trunk and branch (Mueller et al. 2006).

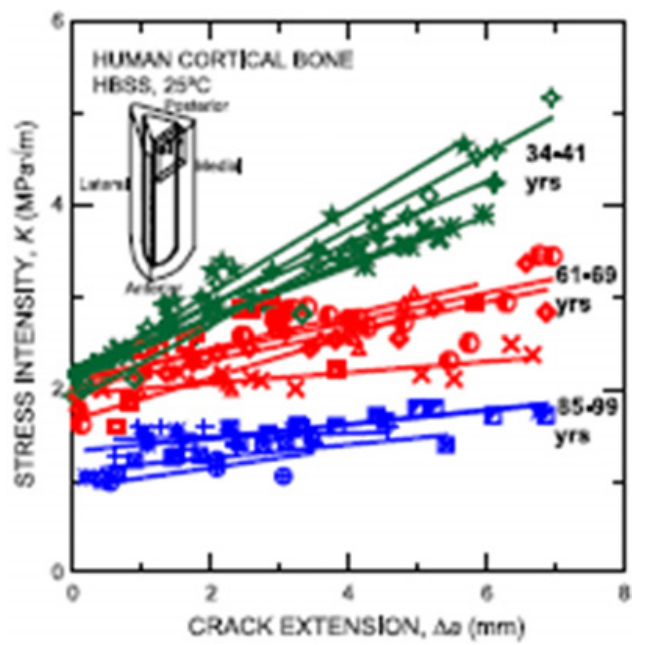

Figure 2. Data from Nalla et al. (2004) showing how fracture toughness increases with crack extension in bone and how this toughening mechanism declines with age.

a long way to go before we can reproduce all the levels of structure found in nature.

But just because this hierarchical structure exists does not mean that it's useful. We are still not sure what the value of this complex structure really is. From the point of view of mechanical properties, the most likely value lies in the improvement of toughness. As Ritchie and colleagues have shown, bone displays R-curve behaviour: its toughness increases with crack extension up to the level of millimetres (see Fig. 2).

Our investigations have shown that the critical distance $\mathrm{L}$, which indicates the level of structure at which the

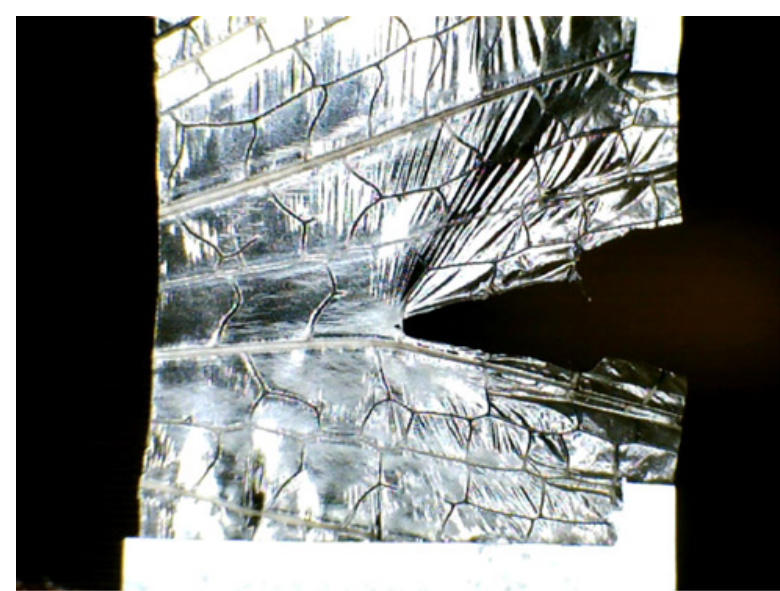

Figure 3. Crack growth in the wing of an insect (locust); veins cause crack tip blunting and re-initiation.

primary toughening mechanism exists, is of the order $0.3-$ $0.4 \mathrm{~mm}$ in bone (Kasiri and Taylor 2008). This corresponds to the spacing of osteons which act as barriers to crack growth and form unbroken ligaments behind the advancing crack. A simpler example is the wing of an insect (Fig. 3), in which periodic veins act as crack-stoppers in a mechanism similar to that seen in aircraft and in "rip-stop" fabrics (Dirks and Taylor 2012).

Poor toughness is a major problem for natural materials, as it is indeed for most non-metallic engineering materials. Most materials fail by cracking, indicating a lack of fracture toughness $\left(\mathrm{K}_{c}\right)$ rather than a lack of strength; all of the stiff materials in nature (e.g. bone, shell, wood, horn) are "quasi-brittle" materials. That is to say, their failure involves a gradual development of damage in the form of microcracks, whose growth is restricted by structural features at different scales. As figure 2 shows, this allows an increase in bone's $\mathrm{K}_{c}$ from about 2 to $5 \mathrm{MPa} \cdot \mathrm{m}^{1 / 2}$. The shells of molluscs and other sea creatures, though they are almost completely made from brittle ceramic materials, increase their $\mathrm{K}_{c}$ considerably by incorporating very thin layers of organic material.

\section{THE BENEFITS OF BEING ALIVE}

The above aspects of materials: their hierarchical micro/nanostructure and the interactions between material and overall component shape, are features which we can expect to incorporate into engineering materials in the future. Indeed we are doing this to some extent already. But there are some other characteristics of nature's materials and structures which are more difficult to replicate and which give nature a real advantage. I am referring to the fact that natural materials are alive. More precisely, they contain living cells which make and regulate the materials in their vicinity, and they benefit from millions of years of evolution leading to improvement by the survival of the fittest. In this section I will briefly review these aspects and attempt to quantify their importance.

Firstly, some (though by no means all) biological materials are able to repair themselves. Bone, for example, 


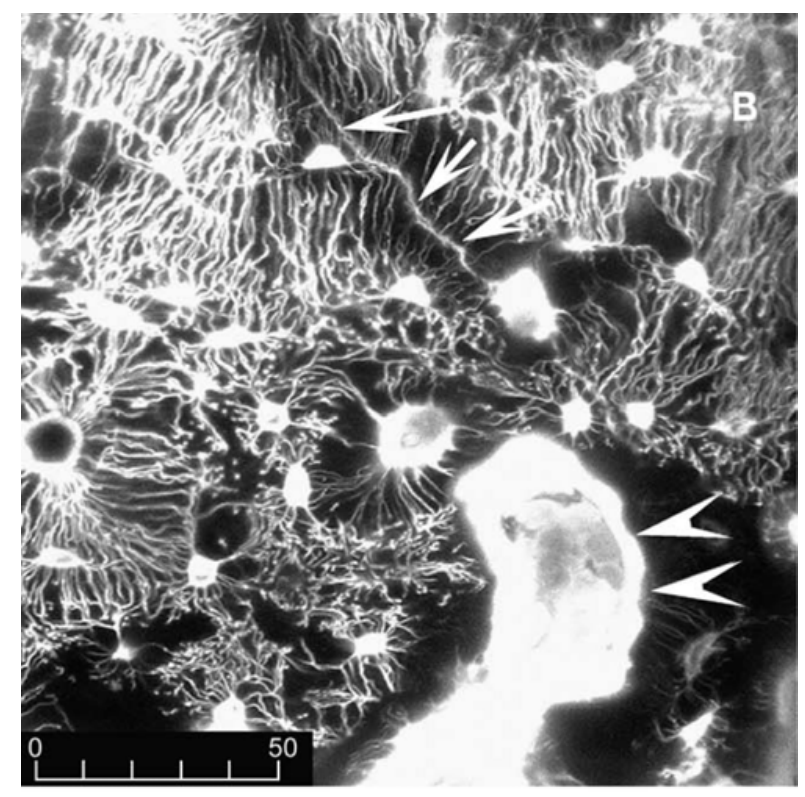

Figure 4. An image from Colopy et al. (2004), showing a section of bone containing a network of osteocytes (small white dots and lines). A fatigue microcrack (top, centre, arrowed) is about to be repaired by a resorption cavity (large white object).

contains cells called osteocytes which are able to detect microcracks when they are about $100 \mu \mathrm{m}$ long and to organise a repair process to remove these cracks before they grow by fatigue. Figure 4 shows an example of this repair in action.

If this repair process didn't happen our bones would fail from fatigue in less than one year. Currently the mechanism whereby this is achieved is not well understood, though it is the subject of much current research by my group and others (Dooley et al. 2012). Recently materials scientists have developed a number of self-repairing materials, including polymers and ceramics which are capable of repairing small cracks. So far these materials can only be made by sacrificing some initial strength, but their self-repair allows them to maintain strength for longer in situations where fatigue failure dominates.

Bones are also capable of changing their shape and structure (e.g. their thickness and density) in response to changes in the loading environment, allowing for a more efficient use of material. This is known as remodelling or adaptation, and acts to refine a shape which has already been gradually improved through evolution. However evolution does not always lead to the best solution. Recently we showed that the legs of arthropods (insects and crabs) had evolved to an optimum shape given the loads upon them; however the same analysis showed that human bones were not optimal (Taylor and Dirks 2012); further shape refinement could almost double the strength of a human femur, for example.

Pain is a very useful signal, indicating that a particular body part is being compromised in some way. Pain in a bone occurs as a result of a crack of the order of a few millimetres in length. In this way you are discouraged from applying load to the bone until the body's cells have time to repair this crack, preventing it from growing to a critical length for brittle fracture.
Finally, there is a feature of the human body and other biological structures which is not strictly a material attribute but which I would like to mention here as an important aspect of the design of structures and components. I refer to reliability: the probability of failure in service. Materials scientists and engineers are constantly being asked to improve the reliability of their materials, which is usually dependant on manufacturing quality, especially the control of defect size. In nature, reliability is low: evidence from primates in the wild suggests that the probability of sustaining a broken bone is $1-3 \%$ over the lifetime of the animal. This would be completely unacceptable in an engineering structure, for which component reliability of the order of one in a million would often be required. The reason that nature can use such low levels of reliability is that the failure of an individual unit (a single animal or person) is not important, compared to the survival of the species.

I have estimated (Taylor 2011) that the advantage gained by nature as a result of some of these living features - specifically the repair of microdamage, pain and low reliability - is a factor of 11.2 on stress. That is to say, nature can use bone at a stress which is more than ten times higher than an engineer would be able to use the same material. This is important to bear in mind when reading about the supposed advantages of natural materials.

\section{CONCLUSIONS}

Materials scientists and engineers have much to learn from studying nature. However, the simple copying of a natural material is unlikely to be useful. Nature uses very different raw materials, and employs them for different purposes, with different objectives. These days, instead of speaking about "Biomimetics", which implies copying from nature, we use the term "Bio-Inspired", indicating that we can use nature as a source of inspiration, a starting point from which to develop new materials, new designs and new manufacturing operations. The results will no doubt look very different from their natural originals - an aircraft doesn't have much in common with a bird - but for a scientist, engineer or designer with an open mind, nature has much to offer.

\section{References}

S. Colopy et al. Rupture of the osteocyte syncytium adjacent to and distant from linear microcracks during adaptation to cyclic fatigue loading. Bone 2004, 881-891. C. Dooley, P. Tisbo T.C. Lee and D. Taylor Rupture of osteocyte processes across microcracks: the effect of crack length and stress. Biomechanics and Modeling in Mechanobiology 2012, 759-766

J.-H. Dirks and D. Taylor Veins improve fracture toughness of insect wings. PLoS ONE 2012: art number e43411

S. Kasiri and D. Taylor A critical distance study of stress concentrations in bone. J. Biomechanics 2008, 603-609

C. Mattheck, Design in Nature: Learning from Trees. 1998, Springer. 
U. Mueller, W. Gindl and G. Jeronimidis. Biomechanics of a branch-stem junction in softwood. Trees 2006, 643-648

R.K. Nalla, J.J. Kruzik, J.H. Kinney and R.O. Ritchie. Effect of aging of the toughness of human cortical bone: evaluation by R-curves. Bone 2004, 1240-1246
D. Taylor, What we can't learn from nature. Materials Science and Engineering C 2011, 1160-1163

D. Taylor D and J.-H. Dirks Shape optimization in exoskeletons and endoskeletons: a biomechanics analysis. Journal of the Royal Society Interface 2012: 3480-3489 